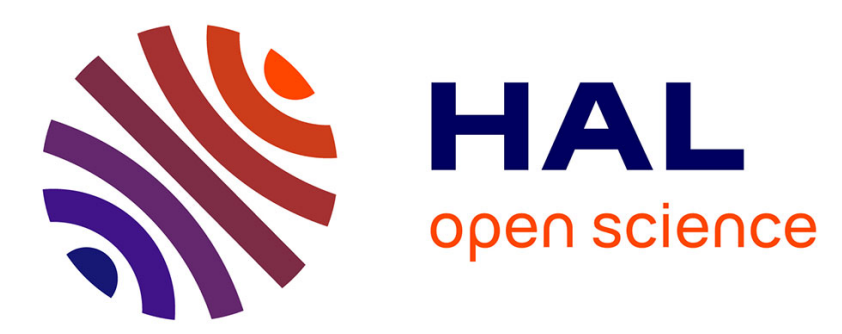

\title{
Initial considerations on the relationship between the optical absorption and the thermal conductivity in dielectrics
}

\author{
C Garca-Segundo, M Villagrán-Muniz, S Muhl, J.-P Connerade
}

\section{To cite this version:}

C Garca-Segundo, M Villagrán-Muniz, S Muhl, J.-P Connerade. Initial considerations on the relationship between the optical absorption and the thermal conductivity in dielectrics. Journal of Physics D: Applied Physics, 2010, 43 (25), pp.255403. 10.1088/0022-3727/43/25/255403 . hal-00569633

\section{HAL Id: hal-00569633 https://hal.science/hal-00569633}

Submitted on 25 Feb 2011

HAL is a multi-disciplinary open access archive for the deposit and dissemination of scientific research documents, whether they are published or not. The documents may come from teaching and research institutions in France or abroad, or from public or private research centers.
L'archive ouverte pluridisciplinaire HAL, est destinée au dépôt et à la diffusion de documents scientifiques de niveau recherche, publiés ou non, émanant des établissements d'enseignement et de recherche français ou étrangers, des laboratoires publics ou privés. 


\title{
Initial considerations on the relationship between the optical absorption and the thermal conductivity in dielectrics
}

\author{
C. García-Segundo ${ }^{1}$, M. Villagrán-Muniz ${ }^{1}$, S. Muhl², J.-P. Connerade ${ }^{3}$ \\ 1. CCADET, Universidad Nacional Autónoma de México (UNAM). Circuito Exterior, \\ Ciudad Universitaria, Apdo. Postal 70-186 C. P. 04510, México, D.F. \\ 2. IIM, Universidad Nacional Autónoma de Mexico (UNAM). Circuito Exterior, \\ Ciudad Universitaria, Apdo. Postal 70-360 y 70-284,C.P. 04510, México, D.F.
}

3. Blackett Laboratory, Imperial College, London SW7 2BZ, UK

Email address: crescencio.garcia@ccadet.unam.mx

The absorption of modulated or pulsed light leads to non-radiative processes that generate a photo-acoustic (PA) wave. The standard model assumes that the amplitude of the PA wave is proportional to the optical absorption. In previous reports we have demonstrated experimentally and theoretically that from this proportionality: 1) one can obtain quantitative measurements of optical properties of dielectric thin films, and 2) that there may exist a relationship between the amplitude of the PA wave and the thermal conductivity of a given sample. Here we present analytic results that clearly show that the optical absorption and the thermal conductivity are coupled to the amplitude of the photoacoustic wave in the modulated regime and that this correlation holds for any type of macroscopic optical and thermal transparency. In particular, the present analysis shows that the product of the optical absorption and the thermal conductivity is related to the PA-amplitude via a partition function.

\section{Introduction}

Photoacoustic absorption spectroscopy is extensively used in several scientific and technical fields. These range from applications in gases, condensed matter, laser control, colloidal materials and the so-called soft-matter [1-6]. Most of the applications related to spectroscopic analysis are focused on either the study of optical properties or the determination of thermodynamic properties. Notably, over the last few years, PA-imaging applications have become of interest. The core of the photoacoustic phenomenon is the generation of 
wideband acoustic-like waves as a consequence of a process of absorption of radiation; the acoustic oscillation is induced in the sample by the interaction with a pulsed or modulated external light source. The range of frequencies of these waves can be from sound to ultrasound with this depending on the time frame of the excitation and on the thermoelastic properties of the medium. As long as the absorption of light is below the saturation limit, the amplitude of the induced sound-like wave is proportional to the amount of absorbed radiant energy. This is an empirical result that has been experimentally verified and accepted by all present PA models [1 3, 5, 7, 9]. However, as Haisch [10] pointed out, the extraction of quantitative information from the PA signals is a non trivial task, with this being true for almost any PA application, and is particular so for PA-spectroscopy. In the PA field, the problem of extracting information has been solved on a case by case basis and this is a considerable limitation for the potential impact of the PA-techniques. One of the main obstacles for achieving quantitative PA measurements is related to the lack of knowledge of the specific amount of energy that is transferred from the optical absorption process into measurable amounts of heat or work. In other words, we do not know the explicit analytic relationship between the optical absorption (optical resistance) and thermal conductivity (thermal resistance). The best we have is the experimental evidence that there exists a rule of proportionality between these resistances; see [11] and references above. Therefore this implies the existence of a specific expression that relates the optical absorption $(\alpha)$ and the thermal conductivity $(\kappa)$ with terms associated with a local change of temperature or pressure. Thus this is an open problem which has yet to be solved.

In the present contribution we focus our attention on setting up conditions for finding a relationship of the type noted above. We start from a consideration of the analysis of solid dielectrics illuminated by low frequency modulated continuous light. Finally, we arrive at results from where one can infer the viability of the quantitative analysis. To do this, we analyse the product of the optical absorption and the thermal conductivity against all physically possible combinations between the macroscopic optical transparency and the thermal transparency. This product appears explicitly in almost the same functional form, in almost all the PA-models. The actual meaning of this product embraces implications beyond the field of photoacoustics alone. We then discuss some aspects of such findings, with the starting point being the general expression used to represent a PA signal for CW-modulated illumination within the visible range. 

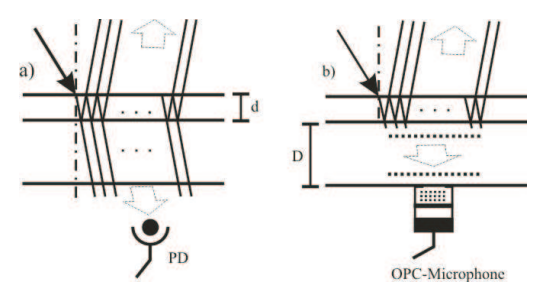

FIG. 1: Schematics of the type of layered system we discuss in the main text. In b) we picture the type of experimental detection we assume for our analysis, and a) is the equivalent optical detection, and is displayed for the purpose of explanatory comparison alone.

For the present case, the modulation frequency is assumed to be in the range of $5 \mathrm{~Hz}$ to $<100 \mathrm{~Hz}$. Then we proceed to analyse, in a case by case manner, the corresponding particular boundary conditions and the material properties. From this we consider the optical absorption and its relationship with the thermal conductivity. The final step is to show the relationship between the rise of temperature within the interaction volume as function of, among other parameters, the optical absorption and the thermal conductivity.

\section{PA theory for modulated illumination}

For simplicity and to aid with the analysis and the presentation of the results, we start from the analysis of a layered solid dielectric. See the figure 1 for schematic guidance. The sample is a solid that is: isotropic, homogeneous and in thermal equilibrium, as required by the standard experimental and theoretical PA model. This model refers to the measurement and analysis of sound-like signals induced in the sample as result of the optical absorption of pulsed or modulated light, whose power is below the saturation limit. Since a fraction of this absorbed optical energy is transferred into the sample via non-radiative processes, there is a local increase of temperature together with a temporary pressure gradient. It should be noted that these non-radiative processes are modulated by the spatial and temporal distribution of the illumination source. The time evolution of this gradient or burst of energy is expressed in terms of sound-like waves: the PA signals. These signals are recorded using a microphone or transducer directly attached to the sample (see figure 1). Experimentally the amplitude of these PA signals are observed to be proportional to the optical absorption, and thus to the intensity of the radiant source.

In the standard PA-model [3, 11, 12] the PA-amplitude output, which we call $H_{0}$, is 
expressed in a general way through a master equation; which can be modified depending on the boundary conditions. This master expression includes the material properties and the instrumental and experimental set-up contributions (such as, the frequency of modulation of the illumination, temperature, humidity, and instrumental response). Once the experiment is set these contributions remain constant during the experimental process, thus there is no restriction to represent these contributions through a constant $G_{0}$. We also recall that the general model assumes that, within a specific spectral distribution, the amplitude of the PA-output is proportional to the intensity of illumination $I_{0}$. Thus the net PA amplitude output is proportional to the product of these parameters, e.g. $H_{0} \propto G_{0} I_{0}$. Similarly, once the experimental conditions have been decided, the intensity of illumination at a given wavelength can be assumed constant. Therefore one can obtain a normalized PA-output, say $H$, by simply taking $H \propto H_{0} /\left(G_{0} I_{0}\right)$. In the reminder of our analysis, any reference to the PA-output we mean the normalized $H$ amplitude, expressed as

$$
H=\frac{r}{r^{2}-1} \frac{2 r-e^{-\alpha z_{0}}\left[(r+1) e^{\sigma z_{0}}+(r-1) e^{-\sigma z_{0}}\right]}{\sigma \kappa\left(e^{\sigma z_{0}}-e^{-\sigma z_{0}}\right)}
$$

The details for the derivation of (1) can be found in the literature [2, 3, 11, 12]. This equation is presented in terms of the thickness of the sample $z_{0}$ (which is also constant for a given experiment), the thermal conductivity $\kappa$ and a parameter $r$, which is the ratio of the optical absorption and the thermal diffusion coefficient, $\sigma=\sqrt{\pi f / a}$; where $f$ is frequency of modulation of the illumination and $a$ is the thermal diffusivity: $a=\frac{\kappa}{\rho c}$. Here, $\rho$ is the sample density and $c$ is the the specific heat at constant pressure $[2,6]$. Then $H$ has units of temperature divided by the flux of power per unit area: ${ }^{\circ} K /\left(\right.$ Watts $\left./ m^{2}\right)$. Thus physically $H$ represents the rate of change of the local temperature as result of the flux of energy from the non-radiative processes; these triggered within a volume of area $A$ and length $z_{0}$. Interestingly, these units are due to the product $\sigma \kappa$ in the denominator of (1). Whilst the remaining terms in (1) produce a dimensionless number that we label as $\xi_{0}$. Therefore, (1) is re-written as $H=\xi_{0} /(\sigma \kappa)$. On the next step we multiply both sides of this expression by $\alpha$. The result is that on the right hand side we have the product $r \xi_{0}$, divided by the product of the optical absorption and the thermal conductivity. Therefore, by defining $\xi=r \xi_{0}$, which is also a dimensionless number, and after re-arranging terms we get 


$$
\kappa \alpha=\frac{\xi}{H}
$$

where $\xi=\frac{r^{2}}{r^{2}-1} \frac{2 r-e^{-\alpha z_{0}}\left[(r+1) e^{\sigma z_{0}}+(r-1) e^{-\sigma z_{0}}\right]}{\left(e^{\sigma z_{0}}-e^{-\sigma z}\right)}$. For given boundary conditions of a given sample, this $\xi$ factor is a constant. Conversely, any change in the material or boundary conditions would imply a change in the value of $\xi$.

Thus, once the amplitude of the waves is normalized as indicated before, the PA amplitude will be expressed according with the many ways as 1 can be reduced; see section 2 for details. An important condition of $H$ is that the optical properties of the sample such as, the reflectance, the optical scattering, the absorbance and the transmittance, are linear functions of the illumination wavelength. We recall that we assume we have a homogeneous and isotropic sample. For means of sensing the information, we assume that the sensor is a membrane microphone, as that of the Open Photoacoustic Cell (OPC) type [4, 13]. Since the absorbed radiant energy induces diffusive phenomena within the sample, then the modulation frequency of the incident illumination must be sufficiently low; this frequency would depend on the material properties. The specific details of these considerations can be found in [3, $13-15]$.

Notice that in [11, we reported a relationship of the type represented by (2), along with experimental support for this description. These results were obtained for only one specific set of boundary conditions. For the present work we further investigate the relationship for the general case.

\section{Different physical conditions}

The various cases covered by the standard PA-models are related to the different material properties and the boundary conditions associated with $\alpha$ and $\sigma$. This follows from the fact that they define the characteristic optical thickness (otherwise known as optical attenuation or optical length, $\mu_{\alpha}=1 / \alpha$ ) and the characteristic thermal thickness (the thermal attenuation or thermal diffusion length, $\left.\mu_{\sigma}=1 / \sigma\right)$, respectively. Therefore, the next stage is to describe the many possibilities in terms of the corresponding boundary conditions along with the respective expressions that are obtained from applying these conditions to the equation (1). The analytic details can be found in $[3,5,4,12$. 


\subsection{Optically Thick (opaque) Materials}

A given material can be optically opaque (optically thick, $\mu_{\alpha}<1$ ), implying that its transmittance is $T=\exp \left(-\alpha z_{0}\right)=0$; and yet it can also be:

\section{1a Thermally thin}

This implies that $\mu_{\sigma} \gg z_{0}$ and $\mu_{\sigma} \gg \mu_{\alpha}$, or that $|r| \gg 1$ and thus $\exp \left( \pm \sigma z_{0}\right) \simeq 1 \pm \sigma z_{0}$; in this case (1) reduces to

$$
H=\frac{1}{\sigma \kappa} \frac{1}{\sinh \left(\sigma z_{0}\right)}
$$

After multiplying both sides of (3) by $\alpha$, and remembering the definition of $r$, the terms can be reorganize to give

$$
\alpha \kappa=\frac{1}{H} \frac{r}{\sinh \left(\sigma z_{0}\right)}
$$

Since $r$ and the sinh are dimensionless, then as expected (4) has units of $\left[\right.$ Watts $\left./\left(m^{2}{ }^{\circ} K\right)\right]$.

\section{1b Thermally thick}

In this case $\mu_{\sigma}<z_{0}$ (or $1<\sigma z_{0}$ ), having $|r|>1$. Here we note that the hyperbolic ratio $1 /\left(e^{x}-e^{-x}\right)$, is $\operatorname{csch}(x)$; which can be represented in terms of an asymptotic series expansion. Using such representation and the respective boundary conditions, then (1) becomes

$$
H=\frac{r}{\left(r^{2}-1\right) \sigma \kappa} e^{-\alpha z_{0}}
$$

After repeating the same procedure used for deriving (4) from (3), we get

$$
\alpha \kappa=\frac{r^{2}}{r^{2}-1} \frac{e^{-\alpha / z_{0}}}{H}
$$

\section{1c Thermally very-thick}

This is where $\mu_{\sigma} \ll z_{0}$ (or more appropriately $1 \ll \sigma z_{0}$ ) and then $|r| \gg 1$. In this case, the argument of the hyperbolic function can be assumed to tend to infinite. With the help of 
the asymptotic representation introduced above, then (1) is reduced to

$$
H=\frac{1}{r} \frac{e^{-\sigma z_{0}}}{\sigma \kappa}
$$

Again after multiplying both sides of equation (7) by $\alpha$ and reorganizing terms we get

$$
\alpha \kappa=\frac{e^{-\alpha z_{0}}}{H}
$$

It should be noted that: 1) the general model only takes into account the thickness of the sample. 2) For the cases considered above, the optical absorption occurs within a volume of area $A$ times a thickness $z_{l}$, which may or may not be the same as the actual sample thickness, $z_{0}$.

\subsection{Optically thin (transparent) materials}

Another set of possibilities arise for samples that are optically thin, $\left(\mu_{\alpha} \gg 1\right)$. This means that its transmittance is $T=\exp \left(-\alpha z_{0}\right) \neq 0$, and therefore, $H$ depends on both: the sample's optical properties and on the thermal properties. Again, an optically thin material can also be

\section{2a Thermally very-thin}

This is for $\mu_{\sigma} \ll z_{0}$ (with $|r|>1$ ), then the equation (1) reduces to

$$
H=\frac{r}{r^{2}-1} \frac{\alpha z_{0}}{\sigma \kappa \sinh \left(\sigma z_{0}\right)}
$$

After using the same procedure as in the previous subsection and noting that at this scale $\sinh \left(\sigma z_{0}\right) \approx \sigma z_{0}+\ldots$, we get

$$
\alpha \kappa=\frac{r^{4}}{\left(r^{2}-1\right) H}
$$

\subsection{Thermally thin}

In this case $\mu_{\sigma}>z_{0}$, and $|r|<1$. Therefore, (1) is reduced to 


$$
H \cong r \frac{e^{-\alpha z_{0}}}{\sigma \kappa}
$$

and correspondingly

$$
\alpha \kappa \cong r^{2} \frac{e^{-\alpha z_{0}}}{H}
$$

\section{2c Thermally thick}

This case refers to $\mu_{\sigma}<z_{0}$ and $|r| \ll 1$, thus from (1) we get

$$
H=r \frac{e^{-\alpha z_{0}}}{\sigma \kappa}
$$

from where we obtain

$$
\alpha \kappa=r^{2} \frac{e^{-\alpha z_{0}}}{H} .
$$

In this way we verify that regardless of how the optical and thermal transparency combine, for each case one can derive an expression which equates with the product $\alpha \kappa$, and in general this is in terms of a dimensionless-type number, $\xi$, divided by the normalised PA-amplitude $H$. This procedure describes in a fundamental way a new approach to the interpretation of the PA experimental data. Moreover, it provides a way to extend the theory, and thus making more viable the quantitative analysis. As indicated above, in [11] we presented results related to this $\alpha \kappa$ product. At that time we considered that there was a possibility that this relationship could lead one to a Widenmann-Franz type law for dielectrics. The present results further support such an idea.

\section{The product $\alpha \kappa$}

In physical terms, the optical absorption coefficient can be seen as a measure of the coupling of each wavelength of the external field with the sample [16]. While within a solid, the transference (coupling) of this optical absorption to thermal energy depends on the sample's ability to conduce heat; and this can be quantified in terms of a flux of free energy [17]. This is explained in more detail in the reminder of the current section. The process of optical to thermal energy transfer involves the generation of collective quanta of mechanic vibrations 
that are defined by the modes of lattice oscillation or lattice waves and can be observed as a spectrum of phononic oscillations; which at the low limit are sound waves [18, 19. In this context, for dielectrics, it has been established that the efficiency of transference of the energy from optical to thermal is indicative of the strength of the interaction of the natural transverse lattice oscillation modes with each wavelength of the external illumination field (this is different to the case for metals where longitudinal waves are involved) [17, 18, 20]. This type of interaction signifies that for each illumination wavelength there exists a specific phonon spectral distribution response (or lattice wave distribution response). In other words, the non-radiative processes, in the sense we discuss here, will occur when at a given wavelength the coupling of the external field with the solid results in inducing specific lattice-vibration modes. These vibrations are responsible for the transport of the energy flux represented by $H$, and thus is what determines the form of the non-radiative absorption spectra; as reported in [5, 11].

On the other hand, we recall that the thermal conductivity is directly related to the phonon mean free path (heat-carriers-free-path) which is determined by phonon-phonon scattering. At room temperature, an isotropic regular solid (crystalline) will have the highest thermal conductivity value when it is pure. Conversely, the thermal conductivity decreases with an increase of the impurities and/or the lattice defect density.

Thus the product $\alpha \kappa$, can be seen as the measure of coherency between the external field and the induced thermal field as result of the matching of the optical frequencies with the phonon mean-free-path distribution; or rather, the coupling between what can be described as the optical impedance to the thermal impedance. We recall that this product has units of $\left[\right.$ Watts $/\left(m^{2} K\right]$. Therefore, this represents a flux of energy per time unit and unit of area, rated by the change of local temperature which only occurs during the presence of the external field (period of illumination). Therefore this flux, as described by this dimensional analysis, bring us to define $\alpha \kappa=z_{l} \rho_{w} / T$. Where $\rho_{w}$ is the volumetric power density: $W /\left(A z_{l}\right) ; T$ is the temperature within the interaction volume. That defined by the illumination area $A$ and the interaction depth, $z_{l}$, that is perpendicular to the surface where the external field is incident. Notice that for an optical transmittance $T_{\text {opt }}=0, z_{l} \neq z_{0}$; while for $T_{o p t} \neq 0, z_{l}=z_{0}$. With consequences as described in the Section 3. Therefore, this volumetric power density can also be written in terms of the time variation of the volumetric energy density as 


$$
\rho_{w}=\frac{d \rho_{e}}{d t}
$$

After considering the $\alpha \kappa$ product, as expressed in the paragraph above, from 15 is imediate that

$$
\frac{d \rho_{e}}{d t}=\frac{\alpha \kappa T}{z_{l}}
$$

The study of the type of interactions we describe here, related to the optical-thermal association and its significance, is not new; as can be seen in Frölich's theory of dielectrics [17]. The action of the external field on a dielectric, assuming one modulation period, is understood to be the amount of external work done on a constant volume, $V=A z_{l}$, of the solid. Since this work, on average, is periodic then it is applied in a cyclic reversible and isothermal way. It is of interest that the Fröhlich interpretation matches with the PA experimental and theoretical descriptions. In PA we assume that the sample is in thermal equilibrium with the environment and interacts with the external field in a reversible way. Therefore, it can be seen that $\rho_{e}$ is the Helmhotlz free energy density, $F / V$; with $F$ being the Helmholtz free energy and $V$ the volume within which the amount of work is done [17]. To the best of our knowledge, this is the first time this interpretation has been proposed. Furthermore we observe that this energy density is related to the partition function $(Z)$ as

$$
\rho_{e}=-k_{B} T \log (Z)
$$

Where $k_{B}$ is the Boltzmann's constant. Here $T$ is referred as the change of temperature within the interacting volume. The relevance of this formalism is that: 1) we have a way to directly connect the description of PA phenomena with the statistical physics theory; 2) and therefore, we can connect the various microscopic phenomena with macroscopic observations; 3) additionally, once we know the partition function for a given sample then one can obtain almost all of the thermodynamic parameters and therefore a quantitative analysis is potentially available [1-3, 5, ,7, 9].

Once we have (17), the sample's internal temperature is the first parameter we can calculate,

$$
T=\frac{z_{l}}{\kappa \alpha} \frac{d \rho_{e}}{d t}
$$


In our description, $\mathrm{Z}$ is related to the spectral distribution of the lattice-vibration modes produced by the absorption of radiation at a specific wavelength. Thus, because the optical absorption is a function of the wavelength then one expects to get different $\mathrm{Z}$ for each wavelength and therefore there will be a change in the thermal conductivity. The corresponding analytic description is complex and for the development of the present study we use only the phenomenon seen in the experimental evidence [11]. In any case, it can be expected that the optical absorption, expressed as induced external work, would cause a change in the local temperature within the interaction volume and that this change is specific for each wavelength. This means that in the interaction of an external field with a solid, the amount of energy that is transferred from optical to thermal is characterized for each wavelength of illumination by an specific phonon distribution (the response to the illumination); and that that response is an attribute of the material at that wavelength.

Now, by combining (15) and (17), we get that

$$
\frac{d \rho_{e}}{d t}=-k_{B}\left[\log (Z) \frac{d T}{d t}+T \frac{d \log (Z)}{d t}\right]=\frac{\kappa \alpha T}{z_{l}} .
$$

On solving (19) two possible physical conditions become apparent: 1 ) when $Z$ is time independent and 2) when $Z$ is time-dependent. We will now consider the significance of these cases. We recall that we assume a homogeneous and isotropic material, and that the frequeny of modulation of the illumination is low. Thus for the time dependant $Z$, we observe that, if at the beginning of a period of illumination we consider an 'instant picture' of the phonon distribution (e.g. lattice-vibration modes) triggered by external field, we would observe a $Z_{o}$ distribution of states. Then if at an arbitrary instant of time later we take a second picture then we would observe that the distribution of states was essentially be the same. This is because the interaction time scale is very short compared to the time scale for the transport and displacement phonon processes. This, combined with the condition that the number of oscillatory components involved is very large, implies that any effective change in the distribution of states will be within the noise range of the average energy distribution due to the ambient temperature and thus undetectable [11, 17, 19]. Since in the right-hand side term within square brackets in (19), $d \log (Z)$ can be written as $d Z / Z$, then after substituting this, the resulting term will be zero. Thus, after arranging terms, we get 


$$
\frac{d T}{T}=-\frac{\kappa \alpha}{z_{l} k_{B} \log (Z)} d t
$$

We solve this for a time, $t$, set within the time frame of illumination in a modulation period, $t_{0}$. During this period, within the volume of interaction, the temperature changes from the initial value $T_{0}$ to a maximum $T+T_{0}$. Thus we solve 20 to get

$$
\log \left(\frac{\delta T+T_{0}}{T_{0}}\right)=-\frac{\kappa \alpha}{k_{B} z_{l} \log (Z)} t_{0} .
$$

From here we obtain that the change of local temperature can be expressed as

$$
\delta T=T_{0}\left\{-1+\exp \left[-\frac{\kappa \alpha}{k_{B} z_{l} \log (Z)} t_{0}\right]\right\} .
$$

This is the burst of temperature that is ultimately the engine for the wave that travels within the sample as a sound-like wave; i.e. the PA-signal. Empirically, from the PA experimental evidence, we know that the local change of temperature is very small compared to the initial temperature: $T_{0} \gg \delta T$. However the caused effects are clearly measurable; as it is done for the PA burst. Note that except the partition function, all the other aspects contained in the PA models are discussed elsewhere in the PA-literature (22] [5, 9, 11]. Thus we explore some consequences of the introduction of this function. In this sense, if instead one chooses to solve 20 for $Z$, we start from 21 and take a series expansion of the exponential function and then we approximate this to first order since this is the most important contributing term at the $T_{0} \gg \delta T$ regime. As result we obtain that

$$
\frac{\delta T}{T_{0}}=-\frac{\kappa \alpha}{k_{B} z_{l} \log (Z)} t_{0}
$$

and after reorganizing terms and solving for $\mathrm{Z}$, we get

$$
Z=\exp \left(-\frac{T_{0} \kappa \alpha}{\delta T k_{B} z_{l}} t_{0}\right)
$$

Let us define $\beta=1 /\left(\delta T k_{B}\right)$, and from 16 we find that, at each illumination wavelength, the term $T_{0} \kappa \alpha t_{0} / z_{l}$ represents the volumetric density of free energy within a single period of illumination. Thus one can re-write (24) as

$$
Z=e^{-\beta \rho_{e}}
$$


recovering 17; which is the definition of $Z$ at the $\delta T \rightarrow 0$ limit. This result implies that the temperature distribution is described by the canonical ensemble.

Now using equation 2 and substituting $\alpha \kappa$ in the equation 24 by the ratio $\xi / H$ we get an expression that is of the same analytic form as 25, albeit this corresponding to the PA phenomena. This function can be associated to a particular sample via the boundary conditions as described in Section 2. Hence, once we know the partition function associated to a sample, then in principle one knows everything about the sample; and one can derive all the observable physical quantities including, as we describe, the optical absorption. It should be noted that the inverse is also true for an optically thin sample: given the absorption spectrum, one can reconstruct the part of the partition function on which the optical absorption depends. However, if the sample is optically thick, then this inverse relationship is no longer unique: it is quite possible that two systems with different partition functions could possess the same optical absorption spectrum. As far as the current analysis goes, this is one of the possible limitations for the present results. However that is subject of future analysis.

Let us assume we know all the terms in $\rho_{e}$, including the initial temperature and the amount of induced change of this temperature. Thus in principle, as referred above, experimentally one can achieve a quantitative reconstruction of the portion of the partition function on which the optical absorption depends; i.e. the amount for which we can normalize the distribution of states at time to calculate e.g. the mean energy, the specific heat, or other thermodynamic parameters.

The other variant that one can obtain from 19 , is that for the partition function being time dependent. This situation arises when conditions are such that the interaction time and the decay times are similar, and the discreteness of the phase space is taken into account [21]. Such possibility emerge e.g. for nano-samples (such as nano-particles, nano-films, and so on). The general feature is that the number of states contributing to the statistical distribution may become in somehow limited and varying with the time. Thus we reorganize terms in 19 and correspondingly we distribute $\log (Z), T, k_{B}$ and the time differential to obtain

$$
\frac{d T}{T}+\frac{d \log (Z)}{\log (Z)}=-\frac{\kappa \alpha}{k_{B} z_{l} \log (Z)} d t .
$$

The PA-experimental evidence indicates if we illuminate the sample for a period of time, say $t_{0}$, then within the interacting volume we will induce a change of local temperature. Going from initial $T_{0}$ to a final value $T=T_{0}+\delta T$. Thus, we integrate (26) to get 


$$
\log \left(\frac{\delta T+T_{0}}{T_{0}}\right)=-\left[\frac{\kappa \alpha}{k_{B} z_{l} \log (Z)} t_{0}+\int \frac{d \log (Z)}{\log (Z)}\right] .
$$

Since we want to solve for the temporal change of temperature, from 27) we obtain that

$$
\delta T=T_{0}\left\{-1+\exp \left[-\frac{\kappa \alpha}{k_{B} z_{l} \log (Z)} t_{0} \int \frac{d \log (Z)}{\log (Z)}\right]\right\} .
$$

Here 28 is somewhat similar to 22 , except for the extra term $\int \frac{d \log (Z)}{\log (Z)}$. Therefore, in this case any macroscopic temperature measurement would produce a noticeable bias temperature field resulting from the variations in the distribution of microstates. In this case, one is immediately tempted to ask: how measurable is the temperature variation? In fact, this will depend on how this temperature variation compares with the fluctuation of the microstates. Further, remembering that $Z$ is a quantity of statistical nature, then the comparison should also be in statistical terms.

What is of interest is that in both regimes covered by (19), a flux of external energy in a confined volume of the sample provokes a local increase of temperature and that this is via non-radiative processes. This phenomenon is the engine that triggers an elasto-mechanical wave which travels within the sample as a sound-like wave; i.e. the photo-induced PA-signal. The analysis for the short time scale, $\tau_{L}$, regime has not been included since it requires an specific and detailed analysis, in view that the theoretical set up is different from the current one, $\tau_{M}$. This is $\tau_{M} \gg \tau_{L}$, and different conditions arise.

\section{Discussion of results}

Up to date, neither, experimentally nor theoretically, is it possible to truly perform quantitative real-time PA-measurements. The best we have is the deterministic process of backtracking the temporal evolution of a process such as the burst of energy occurring in the PA-phenomenon. The current study displays some aspects of this backtracking. In particular this related to describe how the absorbed and thermal energy evolve in time; this is made in terms of the PA-signal representations. From the set of expressions we obtain here there seems to exist a way to perform an effective quantification of the transference of energy, from optical to thermal processes. As we describe, in the modulated illumination regime, the standard model provide us with conditions to obtain the product $\alpha \kappa$, which appears to 
be a consistent result for each case covered by such model. Furthermore, this interpretation bring us to understand that once a dielectric sample is set to interact with a radiation external field, the sample's response to the field is in terms of how this field is coupled to local vibrational modes. If one takes into account the product $\alpha \kappa$, then one can estimate that a measure of such coupling is some how proportional to the thermal conductivity. Then these conditions permit us to define a flux of energy in a similar way as in fluids theory or as described by an electric current. We recall that the current results correspond to the case for low frequency of modulation for the external field. However, the current results are hinting that it could exist similar type of relationship for the short-time scale $\tau_{L}$; such as this with the case for short laser pulses ( $>20 n s)$. Therefore, it would be very much needed to study and understand the implications on the existence of a more general relationship between $\alpha$ and $\kappa$. Specially if we want to know which are the necessary and sufficient conditions for this relationship to exist. By now we consider that regardless the time regime. For the PA phenomena to occur, is required to set up these common features: 1) a flux of external optical energy interacting within a portion of the local volume, that provokes a local increase of temperature; 2 ) the so induced non-radiative processes, would trigger an elasto-mechanical wave which travels within the sample as a sound-like wave [1, 9, 11. This bring us to set the definition and actual physical meaning of the product we analyse in the section 4 . On one hand it is constructed from its interpretation as proportional to a flux of radiative energy, which is work that is made on a solid [17]. As result we arrive to establish that that the relationship between the optical absorption and the thermal conductivity is via a partition function.

\section{Conclusions}

So far the experimental and theoretical interpretations of the PA phenomenon has been well satisfied by a qualitative approach. For general applications one starts by defining the spatial volume within which the optical absorption takes place, and assumes this volume as a heat source $q$, regardless the nature of the source. For practical applications related to condensed matter and gases, this is a good enough. The existence of different models is consequence of the many possible dynamics that the non-radiative process can follow due to many possibilities for boundary conditions and material properties. It should be noticed 
that we have considered all the physically valid combinations between optical and thermal transparency, that occur for illumination at low frequency modulation regime. There is evidence that at short-time scale similar type of results would be obtained. In itself this calls for defining a new set of phase space that equates with the Hamiltonian type of phase space derived form Liouville's theorem (conservation of energy [18, 19]. Our results demonstrates that for any type of sample the PA technique at low-modulation illumination mode, by now, can be used to obtain a complete set of the optical and thermal characteristics of the dielectric sample.

\section{Acknowledgements}

The authors want to acknowledge to the several sponsors thanks to whom this work was performed. In particular CGS wants to thank the support from the Instituto de Ciencia y Tecnología del Distrito Federal, México through a grant contract with CCADET-UNAM. This work was carried on with support also from the National Autonomous University of Mexico through the contracts: IN100706-3 and IN104806-3, DGAPA-UNAM.

[1] A C Tam. Applications of photoacoustic sensing techniques. Rev. Mod. Phys., 58:381-431, 1986.

[2] J A Sell, editor. Photothermal Investigations of Solids and Fluids. Academic Press (London), 1988.

[3] A Rosencwaigh and A Gersho. Theory of the photoacoustic effect with solids. J. Phys. D: Appl.Phys., 47:64, 1976.

[4] M Villagrán-Muniz, C. García-Segundo, H F Ranea-Sandoval, and G M Bilmes. Photoacoustic methods for real-time measurement of laser beam diameter and position, and for laser cavity alignment. Rev. Sci. Instrum., 66:3500, 1995.

[5] C García-Segundo, M Villagrán-Muniz, and S Muhl S. Determination of thin lm optical properties by the photoacoustic opc technique. J. Phys. D: Appl.Phys., 31:165-171, 1998.

[6] S Manohar, A Kharine, J C G van Hespen, W Steenbergen, and T G van Leeuwen. Photoacoustic mammography laboratory prototype: imaging of breast tissue phantoms. J. Biomed. Opt., 9:1172-1181, 2004.

[7] F A McDonald. Generalized theory of the photoacoustic effect mcdonald japplphys v49 p2313 
y1978,. J. Appl. Phys., 49, 1978.

[8] I G Calasso, W Craig, and G J Diebold. Photoacosutic point source. Phys. Rev. Lett., 86:3550-3553, 2001.

[9] C L Cesar, H Vargas, J A Meyer, and L C M Miranda. Photoacoustic effect in solids. Phys. Rev. Lett., 42:1570-1573, 1979.

[10] C Haisch. Quantitative analysis in medicine using photoacoustic tomography. Anal. Bioanal. Chem., 393:473-479, 2009.

[11] C García-Segundo, M Villagrán-Muniz, and S Muhl. On the simultaneous analysis of optical and thermal properties of thin films via pseudo-transmittance spectroscopy: aluminium nitride. J. Phys. D: Appl.Phys., 42:055405, 2009.

[12] M V Marquezini, N Cella, A M Mansanares, H Vargas, and L Miranda. Open photoacoustic cell spectroscopy. Meas. Sci. Technol., 2:396-401, 1991.

[13] P Charpentier, F Lepoutre, and L Bertrand. Photoacoustic measurements of thermal diffusivity description of the drum effect". J. Appl. Phys., 53:608-614, 1982.

[14] A M Mansanares, H Vargas, F Galembeck, J Buijs, and D Bicanic. Photoacoustic characterization of a two-layer system. J. Appl. Phys., 70:7046-7050, 1991.

[15] D D Joseph and L Preziosi. Heat waves. Rev. Mod. Phys., 61:41-73, 1989.

[16] E Fermi. Quantum theory of radiation. Rev. Mod. Phys., 4:87-132, 1932.

[17] H Fröhlich. Theory fo Dielectrics: Dielectric constant and dielectric loss. Monographs on the Physics and Chemistry of Materials. Claredon Press, Oxford, 1949.

[18] J D Walecka, editor. Fundamentals of Statistical mechanics: Manuscript and notes of Felix Bloch. Imperial College Press : World Scientific, 2000.

[19] J M Ziman. Elactrons and Phonons: Theory of transport phenomena in solids. The International series of Monographs in Physics. Claredon Press, Oxford, 1960.

[20] J Bardeen and D Pines. Electron-phonon interaction in metals. Phys. Rev., 99:1140-1150, 1955.

[21] S G Matinyan and B Müller. The partition function in the Wigner-Kirkwood expansion. J. Phys. A: Math. Gen., 39:L285L292, 2006. 